\title{
Study of wave propagation in poroviscoelastic halfspace under normal harmonic load via BEM
}

\author{
Aleksandr Ipatov ${ }^{1, *}$, Svetlana Litvinchuk ${ }^{1}$ \\ ${ }^{1}$ Research Institute for Mechanics, National Research Lobachevsky State University of Nizhni Novgorod, 23, bldg. 6, Prospekt Gagarina \\ (Gagarin Avenue), Nizhny Novgorod, 603950, Russia
}

\begin{abstract}
The present research is dedicated to a problem of a soil dynamics in case when media is subjected to a normal harmonic load. Such type of load may be observed for example because of working wind generator, and the aim is to create a model of possible impact. One of the most appropriate ways for soil modelling is the Biot model of poroelastic media and its enhancements. In this paper we treat a soil as a poroviscoelastic media. Our poroviscoelastic formulation is based on Biot theory of poroelasticity and correspondence principle applied to skeleton of porous material. Standard linear solid model is employed to describe viscoelastic media properties. Boundary integral equations method is applied to solving threedimensional boundary-value problems. The solution of the original problem is constructed in Laplace transforms, with the subsequent application of the algorithm for numerical inversion. To introduce BEdiscretization, we consider the regularized boundary-integral equation. Mixed boundary element discretization is introduced to obtain discrete analogues. Modified Durbin's algorithm of numerical inversion of Laplace transform is used. Research is also dedicated to development of numerical modeling technique based on Boundary Element Method (BEM) in Laplace domain of solution three dimensional poroviscodynamic problems. Numerical example of a poroviscoelastic halfspace under harmonic load is considered. Viscoelastic model parameter influence on dynamic responses of boundary functions is studied. The comparison of transient responses is presented.
\end{abstract}

\section{Introduction}

Wave motion in porous media is an important issue in several branches of engineering science. Mechanics of such advanced materials is relevant to many disciplines, such as geophysics, geo- and biomechanics, seismology, constricting, petroleum engineering. Study of wave propagation processes in saturated porous media originates from Y. I. Frenkel [1] and M. Biot [2-3]. The implementation of the solid viscoelastic effects in the theory of poroelasticity was first introduced by Biot [4]. After that waves in saturated porous continua have been studied by many authors. Common state of the art can be found in works [5-7]. Important, that there is still absent an analytical solution for $3 \mathrm{~d}$ poroelastodynamics and large part of transient response problems can only be solved via numerical methods.

Classical formulations for boundary integral equation (BIE) method with their discretized realization and traditional boundary element method (BEM) are successful approaches for solving three-dimensional problem. Nowadays there are two fundamentally different approaches for constructing such numerical scheme: direct solving in time domain [8-9], or solving in Laplace or Fourier domain [10-11]. Time domain solutions are approximated by a modified Durbin's numerical Laplace transform inversion routine. So the Laplace transform is the main method for solving threedimensional transient problems in porous media. An example problem is solved using the proposed formulation. Modified Durbin's algorithm of numerical inversion of Laplace transform is applied to perform solution in time domain. Durbin [12] developed an approach, namely fast Laplace inverse transform (FLIT) for numerical evaluation of the integrals. Some modifications were recently proposed by Zhao [13] in order to overcome a drawback of constant integration step in FLIT. Different approaches of integration steps constructing are considered. Shanz and Cheng [14] presented an analytical solution in the Laplace domain and analytical time-domain solution without considering viscous coupling effect, and then governing equations was extended for saturated poroviscoelastic media by using the Kelvin-Voigt model and obtained an analytical solution in the Laplace domain for the 1D problem [15].

In present work we modify basic equations for fluidsaturated porous media proposed by Biot by replacing the classical linear elastic model of the solid skeleton with the viscoelastic model. Thus, the new theory can take into account the viscoelastic effect of the solid skeleton. For poroviscoelastic analysis, standard linear solid model is employed.

\section{Problem statement}

We consider a three-dimensional, homogeneous, linear poroviscoelastic solid with volume $\Omega \subset R^{3}$ and 
boundary $\Gamma=\partial \Omega$. The set of differential equations of poroelasticity for displacements $\bar{u}_{i}$ and pore pressure $\bar{p}$ in Laplace domain ( $\mathrm{s}-$ transform parameter) take the following form [5]:

$$
\begin{gathered}
G \bar{u}_{i, j j}+\left(K+\frac{G}{3}\right) \bar{u}_{j, i j}-(\psi-\beta) \bar{p}_{, i}- \\
-s^{2}\left(\rho-\beta \rho_{f}\right) \bar{u}_{i}=-\bar{F}_{i}, \\
\frac{\beta}{s \rho_{f}} \bar{p}_{, i i}-\frac{\phi^{2} s}{R} \bar{p}-(\psi-\beta) s \bar{u}_{i, i}=-\bar{a}, \quad x \in \Omega, \\
\bar{u}^{\prime}(x, s)=\widetilde{u}^{\prime}, \quad x \in \Gamma^{u}, \quad \bar{u}^{\prime}=\left(\bar{u}_{1}, \bar{u}_{2}, \bar{u}_{3}, \bar{p}\right), \\
\bar{t}_{n}^{\prime}(x, s)=\widetilde{t}_{n}^{\prime}, \quad x \in \Gamma^{\sigma}, \quad \bar{t}^{\prime}=\left(\bar{t}_{1}, \bar{t}_{2}, \bar{t}_{3}, \bar{q}\right) .
\end{gathered}
$$

where $\Gamma^{u}$ and $\Gamma^{\sigma}$ denotes boundaries for boundary conditions of $1^{\text {st }}$ and $2^{\text {nd }}$ kind respectively, $G, K$ are elastic moduli, $\phi=V^{f} / V$ is porosity, $F_{i}, \bar{a}$ are bulk body forces.

$$
\begin{gathered}
\beta=\frac{\kappa \rho_{f} \phi^{2} s}{\phi^{2}+s \kappa\left(\rho_{a}+\phi \rho_{f}\right)}, \psi=1-\frac{K}{K_{s}} \\
R=\frac{\phi^{2} K_{f} K_{s}{ }^{2}}{K_{f}\left(K_{s}-K\right)+\phi K_{s}\left(K_{s}-K_{f}\right)}
\end{gathered}
$$

Formulas (3) and (4) describe constants reflecting interaction between skeleton and filler $\kappa$ is permeability. Further, $\rho=\rho_{s}(1-\phi)+\phi \rho_{f} \quad$ is a bulk density, $\rho_{s}, \rho_{a}, \rho_{f}$ are solid, apparent mass density and filler density respectively, $K_{s}, K_{f}$ are elastic bulk moduli of the skeleton and filler respectively. Apparent mass density $\rho_{a}=C \phi \rho_{f}$ was introduced by Biot to describe dynamic interaction between fluid and skeleton. $C$ is a factor depending on the pores geometry and excitation frequency.

Poroviscoelastic solution is obtained from poroelastic solution by means of the elastic-viscoelastic correspondence principle, applied to skeleton's constants $K$ and $G$ in Laplace domain. Forms of functions $K(s)$ and $G(s)$ are depends on chosen viscoelastic model.

\section{Boundary element formulation}

Fundamental and singular solutions are considered in term of singularity isolation. Numerical scheme is based on the Green-Betti-Somigliana formula. Boundary-value problem (1)-(2) can be reduced to the BIE system as follows $[10,16]$ :

$$
\begin{gathered}
\frac{1-\alpha_{\Omega}}{2} v_{i}(\mathbf{x}, s)+ \\
+\int_{\Gamma} T_{i j}(\mathbf{x}, \mathbf{y}, s) v_{i}(\mathbf{y}, s) d \Gamma- \\
-\int_{\Gamma} T_{i k}^{0}(\mathbf{x}, \mathbf{y}, s) v_{i}(\mathbf{x}, s) d \Gamma \\
-\int_{\Gamma} U_{i j}(\mathbf{x}, \mathbf{y}, s) t_{i}(\mathbf{y}, s) d \Gamma=0 \\
\mathbf{x}, \mathbf{y} \in \Gamma
\end{gathered}
$$

where $U_{i j}, T_{i j}$ - fundamental and singular solutions, $T_{i j}^{0}$ contains the isolated singularities, $\mathbf{x} \in \Gamma$ - is an arbitrary point. Coefficient $\alpha_{\Omega}$ equals 1 in case of finite domain and -1 in case of infinite domain.

Boundary surface of our homogeneous solid is discretized by quadrangular and triangular elements and triangular elements are assumed as singular quadrangular elements. We use reference elements: square $\xi=\left(\xi_{1}, \xi_{2}\right) \in[-1,1]^{2}$ and triangle $0 \leq \xi_{1}+\xi_{2} \leq 1$, $\xi_{1} \geq 0, \xi_{2} \geq 0$, and each boundary element is mapped to a reference one by the following formula:

$$
y_{i}(\xi)=\sum_{l=1}^{8} N^{l}(\xi) y_{i}^{\beta(k, l)}, \quad i=1,2,3,
$$

where $l$ - local node number in element $k, \beta(k, l)-$ global node number, $N^{l}(\xi)$ - shape functions. Goldshteyn's displacement-stress mixed model is performed. To discretize the boundary surface eightnode biquadratic quadrilateral elements are used, generalized displacements and tractions - are approximated by linear and constant shape functions, respectively.

Subsequent applying of collocation method leads to the system of linear equations. As the collocation nodes we take the approximation nodes of boundary functions. Gaussian quadrature is used to calculate integrals on regular elements. But if an element contains a singularity, algorithm of singularity avoiding or order reducing is applied. When singularity is excluded we use an adaptive integration algorithm. An appropriate order of Gaussian quadrature is chosen from primarily known necessary precision, if it is impossible, the element is subdivided to smaller elements recursively. Solving the system of linear equations leads to the solution of the initial boundary-value problem in Laplace domain.

\section{Laplace transform inversion}

The poroviscoelastic solution in time domain is obtained with the help of the Laplace transform inversion. Durbin's algorithm [10] is used for numerical inversion of Laplace transform. The inverse Laplace transform is defined as the following contour integral 


$$
L^{-1}\{\bar{f}(\mathbf{x}, s)\}=f(\mathbf{x}, t)=\frac{1}{2 \pi i} \int_{\alpha-i \infty}^{\alpha+i \infty} \bar{f}(\mathbf{x}, s) e^{s t} d s,
$$

where $\alpha>0$ is the arbitrary real constant greater than the real parts of all singularities in $\bar{f}(\mathbf{x}, s)$.

When values of $f(\mathbf{x}, s)$ are available only at the sample points, analytical evaluation of integral in Eq. (7) is impossible. Supposing $s=\alpha+i \omega$ we have the following expressions [6] (for convenience the spatial variable $\mathbf{x}$ is omitted hereinafter)

$$
\begin{gathered}
f(0)=\frac{1}{\pi} \int_{0}^{\infty} \operatorname{Re}[\bar{f}(\alpha+i \omega)] d \omega, \\
f(t)=\frac{e^{\alpha t}}{\pi} \int_{0}^{\infty}\{\operatorname{Re}[\bar{f}(\alpha+i \omega)] \cos \omega t-t>0 . \\
-\operatorname{Im}[\bar{f}(\alpha+i \omega)] \sin \omega t\} d \omega
\end{gathered}
$$

Durbin [12] developed an approach, namely fast Laplace inverse transform (FLIT) for numerical evaluation of the integrals in Eqs. (8) and (9). In this section, we briefly review a modifications recently proposed by Zhao [13] in order to overcome a drawback of constant integration step in FLIT.

Let $R$ be large real number so we can rewrite Eqs. (8) and (9) as follows

$$
\begin{gathered}
f(0)=\frac{1}{\pi} \lim _{R \rightarrow \infty} \int_{0}^{R} \operatorname{Re}[\bar{f}(\alpha+i \omega)] d \omega, \\
f(t)=\frac{e^{\alpha t}}{\pi} \lim _{R \rightarrow \infty} \int_{0}^{R}\{\operatorname{Re}[\bar{f}(\alpha+i \omega)] \cos \omega t, t>0 . \\
-\operatorname{Im}[\bar{f}(\alpha+i \omega)] \sin \omega t d \omega\}
\end{gathered}
$$

Defining the nodes as $0=\omega_{1}<\omega_{2}<\ldots<\omega_{n}<\omega_{n+1}=R$ we approximate Eqs. (10) and (11) as

$$
\begin{gathered}
f(0)=\frac{1}{\pi} \sum_{k=1}^{n} \int_{\omega_{k}}^{\omega_{k+1}} \operatorname{Re}[\bar{f}(\alpha+i \omega)] d \omega, \\
f(t)=\frac{e^{\alpha t}}{\pi} \sum_{k=1}^{n} \int_{\omega_{k}}^{\omega_{k+1}}\{\operatorname{Re}[\bar{f}(\alpha+i \omega)] \cos (\omega t)-t>0 . \\
-\operatorname{Im}[\bar{f}(\alpha+i \omega)] \sin (\omega t)\} d \omega
\end{gathered}
$$

In each segment $\left[\omega_{k}, \omega_{k+1}\right], k=\overline{1, n}$, the real and imaginary parts of $\bar{f}(s)$ are approximated with linear functions as follows

$$
\begin{gathered}
\operatorname{Re}[\bar{f}(\alpha+i \omega)] \approx F_{k}+\frac{F_{k+1}-F_{k}}{\omega_{k+1}-\omega_{k}}\left(\omega-\omega_{k}\right), \\
\operatorname{Im}[\bar{f}(\alpha+i \omega)] \approx G_{k}+\frac{G_{k+1}-G_{k}}{\omega_{k+1}-\omega_{k}}\left(\omega-\omega_{k}\right),
\end{gathered}
$$

where $F_{k}=\operatorname{Re}\left[\bar{f}\left(\alpha+i \omega_{k}\right)\right], \quad G_{k}=\operatorname{Im}\left[\bar{f}\left(\alpha+i \omega_{k}\right)\right]$.

Substituting Eq. (14) and (15) into Eqs. (12) and (13) and making direct integration we obtain

$$
f(0) \approx \sum_{k=1}^{n}\left[\frac{\left(F_{k+1}-F_{k}\right) \Delta_{k}}{2 \pi}\right]
$$

$$
\begin{aligned}
& f(t) \approx \frac{e^{\alpha t}}{\pi t^{2}} \sum_{k=1}^{n}\left[\frac{F_{k+1}-F_{k}}{\Delta_{k}}\left(\cos \left(\omega_{k+1} t\right)-\cos \left(\omega_{k} t\right)\right)+\right. \\
& \left.\frac{G_{k+1}-G_{k}}{\Delta_{k}}\left(\sin \left(\omega_{k+1} t\right)-\sin \left(\omega_{k} t\right)\right)\right],
\end{aligned}
$$

where $t>0, \Delta_{k}=\omega_{k+1}-\omega_{k}$.

\section{Test example}

In order to assess the accuracy of the proposed BEM formulation the following problem is solved (see Fig. 1): three-dimensional anisotropic elastic prismatic body is clamped at its left end, and subjected to uniaxial and uniform impact loading $t_{2}=t_{2}^{*} H(t), t_{2}^{*}=-1 \mathrm{~N} / \mathrm{m}^{2}$ at the right end, $H(t)$ is a Heaviside step function. The remaining surfaces are traction free. Poroelastic material parameters are:

$$
\begin{array}{lr}
K=8 \cdot 10^{9} \mathrm{~N} / \mathrm{m}^{2}, & G=6 \cdot 10^{9} \mathrm{~N} / \mathrm{m}^{2}, \phi=0.66, \\
\rho=2458 \mathrm{~kg} / \mathrm{m}^{3}, & K_{s}=3.6 \cdot 10^{10} \mathrm{~N} / \mathrm{m}^{2}, \\
\rho_{f}=1000 \mathrm{~kg} / \mathrm{m}^{3}, & K_{f}=3.3 \cdot 10^{9} \mathrm{~N} / \mathrm{m}^{2}, \\
k=1.9 \cdot 10^{-10} \mathrm{~m}^{4} /(N \cdot s), &
\end{array}
$$

In this example, we employ the following relation to produce the integration steps $\Delta_{k}=\omega_{k+1}-\omega_{k}$ in both Zhao's algorithms:

$$
\omega_{k}=e^{(k x)^{m}}-1,
$$

where $\quad m=0.8, \quad k=1,2, \ldots, 480 \quad$ and $x=\left(\ln \left(\omega_{\max }+1\right)\right)^{1 / m} / k$, with $\omega_{\max }=300$.

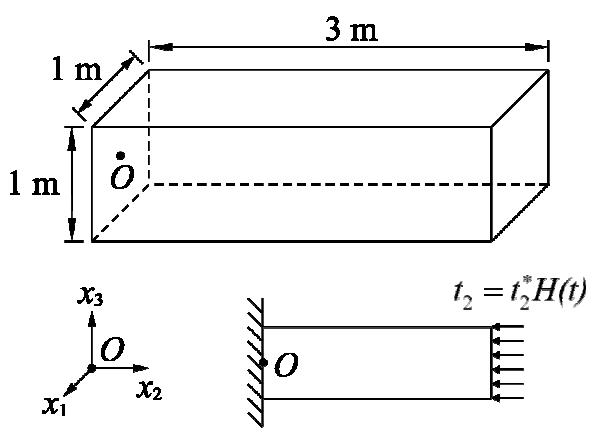

Fig. 1. Convergence with the BEM mesh refinement.

Figures 2 and 3 show the displacement $u_{2}(t)$ at the center point of the loaded end and tractions $t_{2}(t)$ at the center point of the clamped end. 


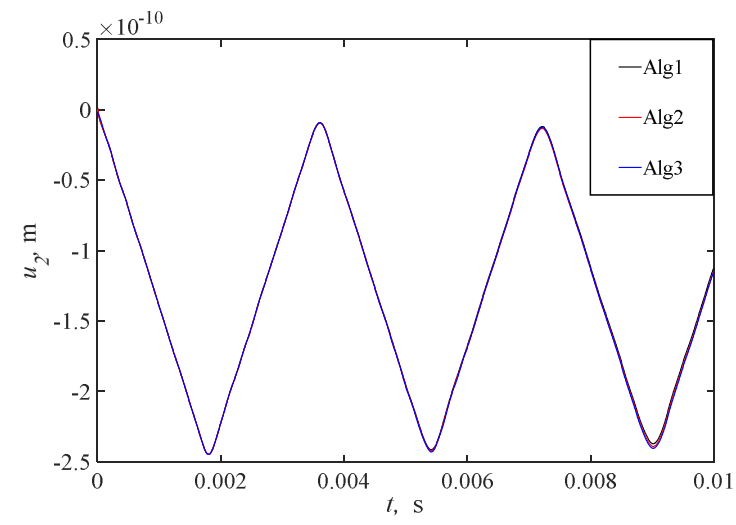

Fig. 2. Displacements $u_{2}$ in case of different Laplace transform inversion algorithms.

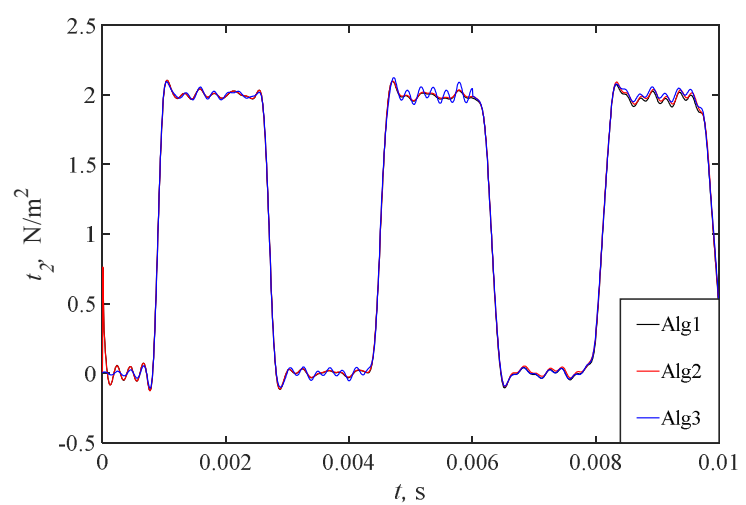

Fig. 3. Tractions $t_{2}$ in case of different Laplace transform inversion algorithms.

Standard linear solid model:

$$
\begin{gathered}
\bar{K}(s)=K^{\infty} \cdot\left[(\beta-1) \frac{s}{s+\gamma}+1\right] \\
\bar{G}(s)=G^{\infty} \cdot\left[(\beta-1) \frac{s}{s+\gamma}+1\right] \\
\beta=K^{0} / K^{\infty}=G^{0} / G^{\infty}
\end{gathered}
$$

Parameters of standard model : $\beta=4, \gamma=1000,100$ and 10.

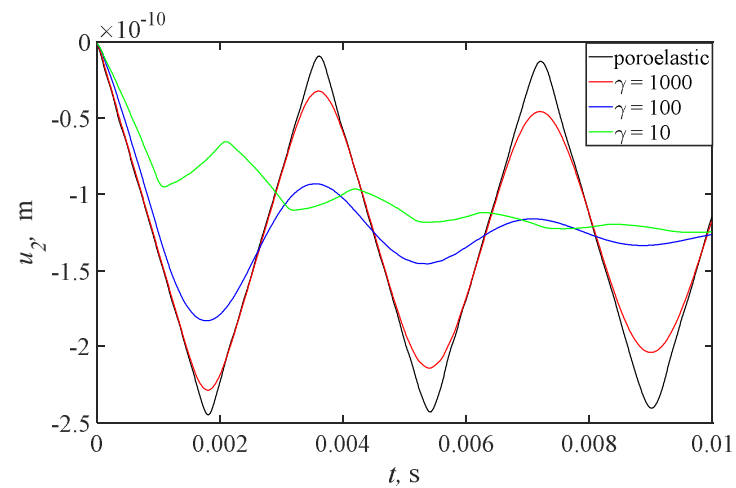

Fig. 4. Displacements $\mathrm{u}_{2}$ in case of standard linear solid model.

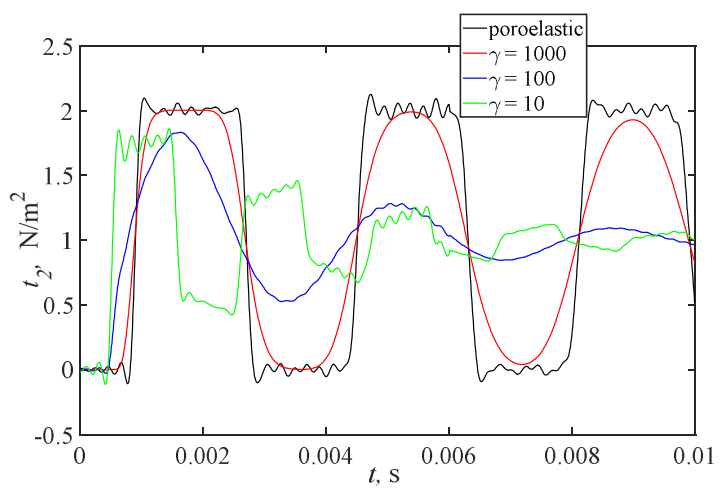

Fig. 5. Tractions $t_{2}$ in case of standard linear solid model.

\section{Numerical example}

Problem of a vertical load on a slab bonded on a halfspace is considered (Fig.6).

Poroelastic material parameters are:

$$
\begin{array}{lr}
K=8 \cdot 10^{9} \mathrm{~N} / \mathrm{m}^{2}, & G=6 \cdot 10^{9} \mathrm{~N} / \mathrm{m}^{2}, \phi=0.66, \\
\rho=2458 \mathrm{~kg} / \mathrm{m}^{3}, & K_{s}=3.6 \cdot 10^{10} \mathrm{~N} / \mathrm{m}^{2}, \\
\rho_{f}=1000 \mathrm{~kg} / \mathrm{m}^{3}, & K_{f}=3.3 \cdot 10^{9} \mathrm{~N} / \mathrm{m}^{2},
\end{array}
$$$$
k=1.9 \cdot 10^{-10} \mathrm{~m}^{4} /(N \cdot s)
$$

that corresponds to Berea sandstone [5]. The reference point $\mathrm{P}(10 ; 0 ; 0)$ is situated on a surface of a halfspace.

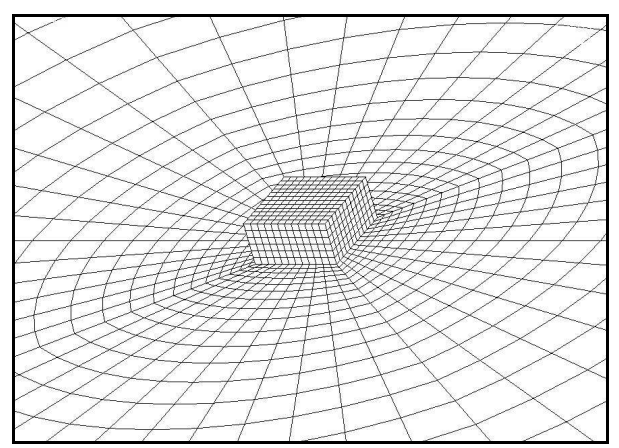

Fig. 6. BE mesh visualisation.

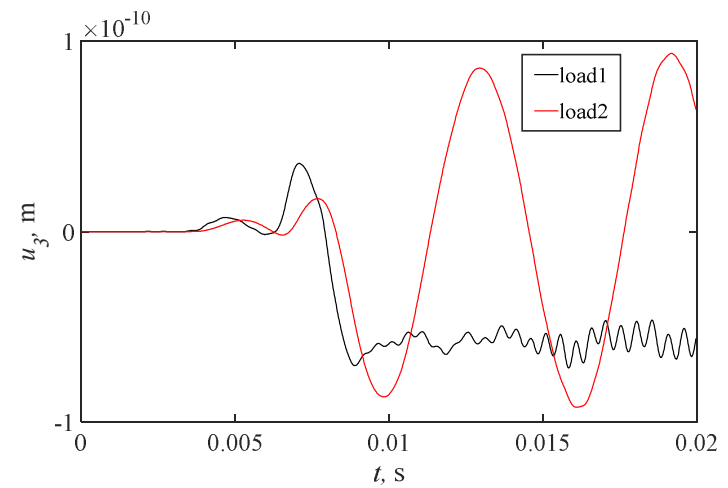

Fig. 7. Displacements $u_{3}$ in case of different load types. 


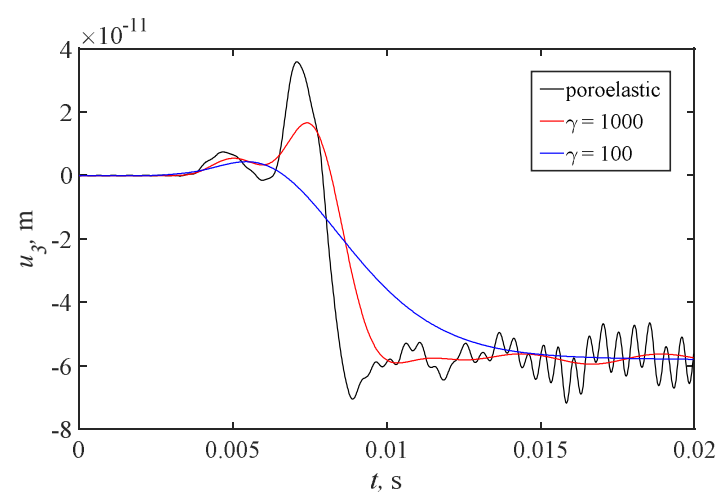

Fig. 8. Displacements $u_{3}$ in case of standard linear solid model (Heaviside-type load).

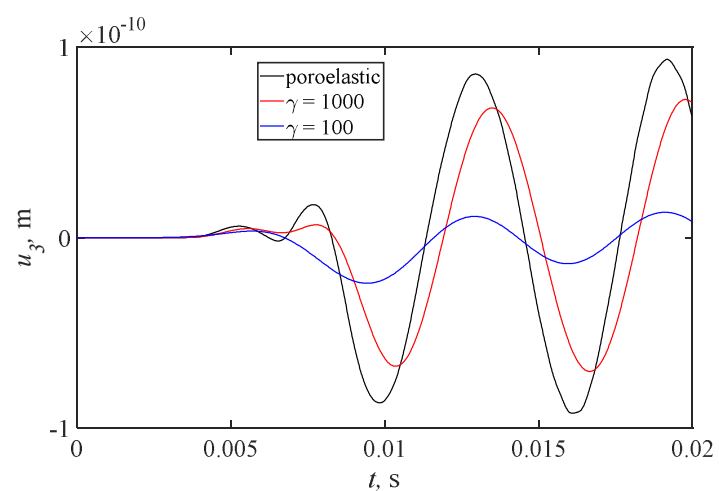

Fig. 9. Displacements $u_{3}$ in case of standard linear solid model (Harmonic load).

\section{Concluding remarks}

A Laplace domain BEM formulation based on integral representations of the fundamental solutions has been presented for the analysis of three-dimensional anisotropic elastodynamic problems. The modified Durbin's method we used to invert solution to the time domain is proved to be an accurate and efficient method particularly well suited for the dynamic problems of the linear poroviscoelasticity. Boundary integral equations method and boundary element method are applied in order to solve three dimensional boundary-value problems. The poroviscoelastic media modelling is based on Biot's theory of porous material in combination with the elastic-viscoelastic corresponding principle. Viscous properties are described by model with weakly singular kernel.

A problem of Heaviside-type and harmonic vertical load acting on a slab bonded on a poroviscoelastic halfspace is solved. An influence of viscoelastic parameter on Rayleigh wave response is demonstrated.

This work was supported by a grant from the Government of the Russian Federation (contract No. 14.Y26.31.0031).

\section{References}

1. J. Frenkel, Journal of Physics, 8, 230 (1944).

2. M. A. Biot, J. Acoust. Soc. Am., 12, 155 (1941)

3. M. A. Biot, J. Acoust. Soc. Am., 2, 182 (1955)

4. M. A. Biot, J. Acoust. Soc. Am., 27(5). 459 (1956)
5. M. Schanz, Wave Propagation in Viscoelastic and Poroelastic Continua, Springer, Berlin (2001)

6. R. de Boer, Appl. Mech. Rev., 49(4), 201 (1996)

7. V. N. Nikolaevskiy, J. Eng. Mech. 131(9), 888 (2005)

8. D. Nardini, C. A. Brebbia, Boundary Element Methods in Engineering, C. A. Brebbia (Ed.), Springer, Berlin (1982)

9. W. J. Mansur, A Time-Stepping Technique to Solve Wave Propagation Problems Using the Boundary Element Method, $\mathrm{PhD}$ thesis, University of Southampton (1983)

10. T. A. Cruze, F. J. Rizzo, J. Math. Anal. Appl., 22(1), 244 (1968)

11. V. A. Babeshko, Proceedings of the USSR Academy of Sciences, 284, 73 (1985)

12. F. Durbin Comput. J. 17, 371 (1974)

13. X. Zhao Int. J. Solids Struct. 41, 3653(2004)

14. M. Schanz, A.H.D. Cheng, Acta Mech. 145, 1 (2000)

15. M. Schanz, A.H.D. Cheng, J Appl Mech., 68, 192 (2001)

16. A.G. Ugodchikov, N.M. Hutoryanskii, Boundary element method in deformable solid mechanics, Kazan State University, Kazan. (1986)

17. A.A. Belov, L.A. Igumnov, A.N. Petrov Probl. Strength Plast., 79(4), 425 (2017)

18. A.A. Ipatov, L.A.Igumnov, A.A. Belov Springer Proceedings in Physics, 193, 331 (2017)

19. L.A. Igumnov, S.Yu. Litvinchuk, I.P. Markov Materials Physics and Mechanics 28, 86 (2016) 
